\title{
Low Complexity HEVC Encoder for Visual Sensor Networks
}

\author{
Zhaoqing Pan ${ }^{1,2, *}$, Liming Chen ${ }^{1}$ and Xingming Sun ${ }^{1}$ \\ Received: 26 October 2015; Accepted: 26 November 2015; Published: 2 December 2015 \\ Academic Editor: Leonhard M. Reindl \\ 1 School of Computer and Software, Jiangsu Engineering Center of Network Monitoring, \\ Nanjing University of Information Science and Technology, Nanjing 210044, China; \\ leegend_chen@outlook.com (L.C.); sunnudt@163.com (X.S.) \\ 2 School of Computer Science and Engineering, Hebei University of Technology, Tianjin 300401, China \\ * Correspondence: zqpan3-c@my.cityu.edu.hk; Tel.: +86-25-5873-1426
}

\begin{abstract}
Visual sensor networks (VSNs) can be widely applied in security surveillance, environmental monitoring, smart rooms, etc. However, with the increased number of camera nodes in VSNs, the volume of the visual information data increases significantly, which becomes a challenge for storage, processing and transmitting the visual data. The state-of-the-art video compression standard, high efficiency video coding (HEVC), can effectively compress the raw visual data, while the higher compression rate comes at the cost of heavy computational complexity. Hence, reducing the encoding complexity becomes vital for the HEVC encoder to be used in VSNs. In this paper, we propose a fast coding unit (CU) depth decision method to reduce the encoding complexity of the HEVC encoder for VSNs. Firstly, the content property of the CU is analyzed. Then, an early CU depth decision method and a low complexity distortion calculation method are proposed for the CUs with homogenous content. Experimental results show that the proposed method achieves $71.91 \%$ on average encoding time savings for the HEVC encoder for VSNs.
\end{abstract}

Keywords: visual sensor networks; video compression; HEVC; coding unit; low complexity

\section{Introduction}

Visual sensor networks (VSNs) have emerged in response to the developments in image sensor technology, sensor networking and distributed computing, which have been widely used in security surveillance, environmental monitoring, smart rooms, and so on [1,2]. VSNs consist of a number of visual sensor nodes named camera nodes, which are used to collect visual information. However, the visual sensors generate a huge amount of visual data as compared to the traditional scalar sensors [3,4]. This becomes a challenge for storage, processing and transmitting the visual data due to the current storage, computing and transmission capability being still limited. Hence, the high compression rate and low complexity are the key requirements of VSNs [5,6]. High efficiency video coding (HEVC) $[7,8]$ is the state-of-the-art video compression standard, which can efficiently compress the raw visual data. The compression rate of HEVC is twice as much as the former video compression standard, H.264 $[9,10]$. However, the achieved compression rate is at the cost of the heavy computational complexity of a series of advanced coding tools used in the HEVC, such as the quadtree-based coding unit (CU), the rate distortion optimization (RDO) technique, and so on. Hence, reducing the encoding complexity becomes vital for the HEVC encoder to be used in VSNs.

To reduce the encoding complexity of the HEVC encoder, many researchers have devoted their efforts to optimizing the HEVC encoding process [11,12]. In [11], Choi et al. proposed an early termination for the $\mathrm{CU}$ size decision process, in which the $\mathrm{CU}$ size decision process is terminated if the current CU selects the Merge/Skip mode as its best prediction unit mode. In [13], Pan et al. proposed a fast $\mathrm{CU}$ depth decision method by using the $\mathrm{CU}$ depth selection correlation between 
the current CU and its spatiotemporal neighboring CUs. In [14], Shi et al. proposed a fast CU size decision method based on adaptive $C U$ depth selection, in which an adaptive $C U$ depth set is derived by using the encoding information of the previously encoded frames. In [15], Zhang et al. proposed a machine learning-based coding unit depth decision for flexible complexity allocation in HEVC. Based on the spatiotemporal encoding parameters of the HEVC encoder, Ahn et al. proposed a fast CU encoding method, which consists of an early CU Skip mode decision method and a fast CU size decision method [16]. Based on the rate distortion differences between the root $\mathrm{CU}$ and children CUs, Goswami et al. proposed an early termination method for the CU depth decision process [17]. Based on the texture complexity of the video content, Tian et al. proposed an adaptive prediction unit mode decision for the HEVC intra-coding [18]. In [19], Kim et al. proposed a fast Skip mode decision method based on the rate distortion optimization for HEVC. According to the differential motion vector and coded block flag, an early determination of the prediction unit mode decision for HEVC was proposed in [20]. In [21], Lee et al. proposed an early Skip mode decision for the HEVC encoder by utilizing the rate distortion characteristics of the Merge mode. By considering the motion activity and hierarchical depth correlation, Pan et al. proposed an early Merge/Skip decision method for the low complexity HEVC encoder [12]. These methods can efficiently remove the encoding complexity of the HEVC encoder for universal video coding. However, the content characteristics of the videos that are generated by the visual sensor camera are not considered, and the encoding complexity can be further improved by considering the content property of the visual sensor videos.

In this paper, we propose a low complexity HEVC encoder for VSNs by optimizing the CU size decision process. The rest of this paper is organized as follows. Section 2 presents the motivations and statistical analyses. Section 3 introduces the details of the proposed fast $C U$ size decision method. Experimental results are given in Section 4 . At last, Section 5 concludes this paper.

\section{Motivations and Statistical Analyses}

When compressing the raw videos that are generated by the visual sensor camera, the videos are separated into images/frames, then the images/frames are encoded one by one. Each frame is split into slices; the slices are further partitioned into a group of coding tree units (CTUs), which are the basic processing units of the HEVC encoder. Based on the quadtree, the CTUs are further split into CUs. In order to achieve the maximum compression rate, HEVC supports flexible CU sizes from $64 \times 64$ to $8 \times 8$, which corresponds to the CU quadtree Depth 0 to Depth 3 . In the CU encoding process, the HEVC encoder checks $4^{n}, n \in[0,1,2,3]$, CU partitions for each quadtree depth level $n$, which totally equals $4^{0}+4^{1}+4^{2}+4^{3}=85 \mathrm{CU}$ partitions. For the $\mathrm{CU}$ intra-/inter-prediction, a CU is further partitioned into one, two or four prediction units (PUs) according to the prediction type, and the $\mathrm{PU}$ is the basic processing unit of the intra-/inter-prediction. To remove the spatial and temporal redundancy, the HEVC encoder supports $11 \mathrm{PU}$ modes, including Merge/Skip mode, eight inter-PU modes (i.e., inter- $2 \mathrm{~N} \times 2 \mathrm{~N}$, inter- $2 \mathrm{~N} \times \mathrm{N}$, inter- $\mathrm{N} \times 2 \mathrm{~N}$, inter- $\mathrm{N} \times \mathrm{N}$, inter- $2 \mathrm{~N} \times \mathrm{nU}$, inter- $2 \mathrm{~N} \times \mathrm{nD}$, inter-nL $\times 2 \mathrm{~N}$ and inter-nR $\times 2 \mathrm{~N}$.) and two intra-PU modes (i.e., intra- $2 \mathrm{~N} \times 2 \mathrm{~N}$ and intra-N $\times \mathrm{N}$.). For CU encoding, these $11 \mathrm{PU}$ modes are checked sequentially. Figure 1 shows an example of the quadtree-based CUs and their PU modes. Ultimately, the best CU quadtree depth level, $d^{*}$, and the best PU mode, $p^{*}$, are determined according to the minimization of the Lagrangian cost function [22],

$$
\left\{\begin{array}{l}
\left\{d^{*}, p^{*}\right\}=\arg \min _{d \in \mathbf{D}}\left(\sum \arg \min _{p \in \mathbf{P}} J(\boldsymbol{O}, \boldsymbol{C}(d, p))\right) \\
J(\boldsymbol{O}, \boldsymbol{C}(d, p))=S S D(\boldsymbol{O}, \boldsymbol{C}(d, p))+\lambda \cdot R(d, p)
\end{array}\right.
$$

where $\mathbf{D}$ is the candidate set of the CU quadtree depth levels and $\mathbf{D} \in\{0,1,2,3\} ; \mathbf{P}$ indicates the candidate set of the $11 \mathrm{PU}$ modes; $J$ is the rate distortion cost function; $O$ is the original CU; and $C$ denotes the reconstructed $\mathrm{CU}$, which is achieved by encoding the original $\mathrm{CU} \boldsymbol{O}$ with the $\mathrm{CU}$ quadtree depth level $d$ and PU mode $p$; the $\operatorname{SSD}(d, p)$ denotes the sum of squared differences (SSD) between the original CU $\boldsymbol{O}$ and its reconstructed CU $C ; \lambda$ is the Lagrangian multiplier; $R(d, p)$ represents the 
number of total bits for encoding this CU, which is obtained by a table lookup. This "try all and select the best" CU quadtree depth level decision and PU mode selection method efficiently improves the coding efficiency of the HEVC encoder; however, it also results in heavy computation load and limits the use of the HEVC encoder in VSNs.

In the $\mathrm{CU}$ encoding process, only parts of the $\mathrm{CU}$ partitions are finally chosen as the best $\mathrm{CU}$ partitions, which are from 1 to 64 ; " 1 " represents that the CU quadtree depth Level 0 is selected as the best depth level; "64" indicates the CU quadtree depth Level 3 is chosen as the optimal depth level. To analyze the best CU quadtree depth level distribution, three different HD visual sensor video sequences with a resolution of $1280 \times 720$, including "Fourpeople", "Johnny" and "Vidyo1", are tested. The HEVC reference software HM12.0 [23] is used as the software platform. The test conditions are listed as follows: the maximum CU size is $64 \times 64$, and the maximum CU quadtree depth is four; the fast motion estimation method is TZSearch, and the search range equals [-64, 64]; four quantization parameters (QPs) are adopted. The statistical results are tabulated in Table 1.

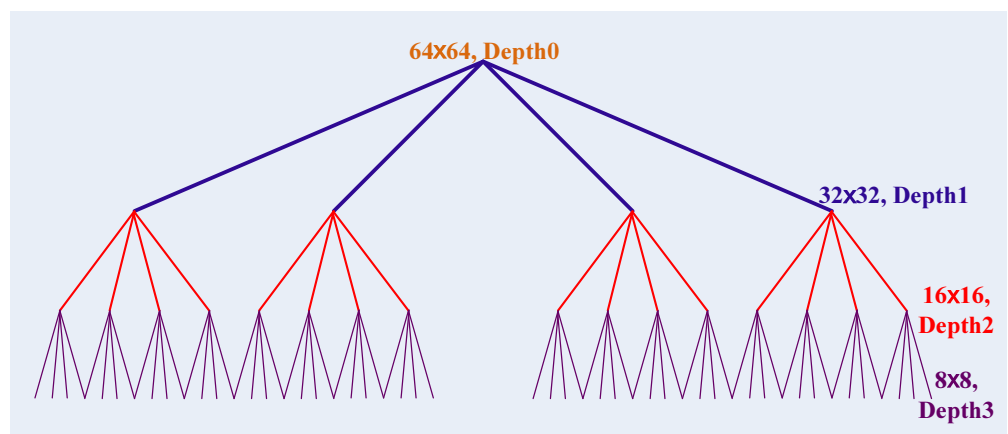

(a)

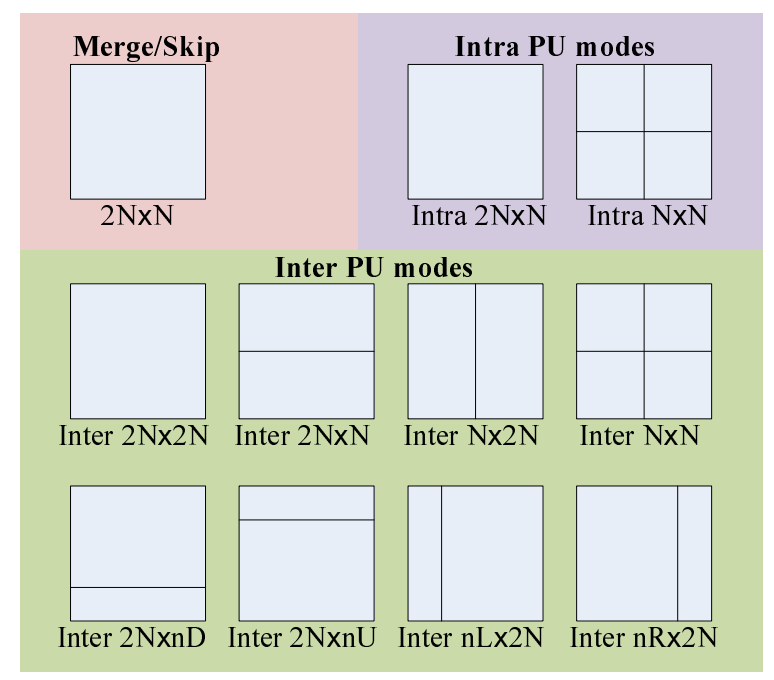

(b)

Figure 1. The quadtree-based coding units (CUs) and their prediction unit (PU) modes. (a) HEVC CU quadtree partition process; the maximum CU size $=64 \times 64$, the maximum quadtree depth $=4 ;(\mathrm{b})$ all prediction unit modes for a CU.

From Table 1, it can be observed that there are $68.26 \%, 16.07 \%, 14.33 \%$ and $1.34 \%$ CUs selecting the quadtree depth Levels 0, 1, 2 and 3 as their best CU quadtree depth level, respectively. In addition, the probability decreases as the depth level increases and as the QP decreases. This is because for the video that is generated by the visual sensor camera, it contains a huge number of regions with simple content, such as the background, and these regions are quite suitable for encoding in a large CU 
size. Moreover, more prediction residuals are transformed and quantized into zeros as QP increases, which results in the video content becoming simple, and more CUs select the large CU size as their best depth level. On the other hand, the CUs with depth Level 0 hold the largest proportion, and the number of CUs with depth Level 3 is quite small. Therefore, if the best quadtree depth level of a CU is determined early, significant encoding time could be saved.

Table 1. Statistical results of the best CU quadtree depth level distribution (\%). QP, quantization parameter.

\begin{tabular}{cccccc}
\hline Sequence & QP & Level 0 & Level 1 & Level 2 & Level 3 \\
\hline FourPeople & 22 & 49.48 & 24.79 & 22.13 & 3.60 \\
& 27 & 66.59 & 16.50 & 15.19 & 1.71 \\
& 32 & 74.69 & 11.88 & 12.46 & 0.98 \\
& 37 & 79.57 & 8.78 & 11.15 & 0.50 \\
\hline Johnny & 22 & 51.30 & 26.48 & 19.91 & 2.31 \\
& 27 & 68.30 & 17.63 & 13.26 & 0.81 \\
& 32 & 76.74 & 11.87 & 10.95 & 0.44 \\
& 37 & 82.57 & 7.58 & 9.59 & 0.25 \\
\hline Vidyo1 & 22 & 48.94 & 26.55 & 21.07 & 3.44 \\
& 27 & 65.94 & 18.27 & 14.51 & 1.27 \\
& 32 & 74.66 & 13.12 & 11.66 & 0.55 \\
& 37 & 80.34 & 9.35 & 10.07 & 0.24 \\
\hline Average & & 68.26 & 16.07 & 14.33 & 1.34 \\
\hline
\end{tabular}

\section{The Proposed Low Complexity HEVC Encoder for VSNs}

\subsection{The Proposed All-Zero Block-Based Fast CU Depth Decision Method}

The videos that are captured by the visual sensor camera contain a huge number of homogenous regions, such as the background; for these regions, they are quite suitable for encoding in a large CU partition size due to a large-sized CU being able to represent the prediction residual in a small number of symbols than is possible in the case of several small-sized CUs [24]. In addition, the inter-prediction residuals of these regions have a large probability to be transformed and quantized to zeros [25-27]. Therefore, it is reasonable to design an early termination for the CU size decision process based on the quantized coefficients. In video coding, the CU for which its inter-prediction/intra-prediction residual is transformed and quantized to zeros is called the all-zero block (AZB). To exploit the relationship between the AZB and the best CU depth selection, the event $\mathrm{A}$ indicates after encoding the CUs of depth level $i$ with the Megre/Skip mode and inter-2N $\times 2 \mathrm{~N}$ PU mode; they are AZBs. The event $\mathbf{B}$ represents that the CU quadtree depth $i$ is chosen as the best $C U$ depth level; the conditional probability $\mathrm{P}(\mathbf{B} \mid \mathbf{A})$ is analyzed. The statistical results are shown in Figure 2.

From Figure 2, it can be seen that when the CUs with quadtree depth $i$ are AZBs, the CU has a rather large probability to select the quadtree depth level $i$ as its optimal depth level. The conditional probability of $P(\mathbf{B} \mid \mathbf{A})$ is from $78.34 \%$ to $91.82 \%, 86.63 \%$ on average. It also can be observed that the probability of $P(\mathbf{B} \mid \mathbf{A})$ increases as the QP value becomes large; this is because the large QP makes the encoding content become simple and homogenous, which results in more prediction redials being quantized to zeros. Hence, based on the above analyses, the optimal CU quadtree depth level decision process is terminated early if:

$$
\sum_{i} Q_{\text {Merge }_{i}}+\sum_{i} Q_{\text {Inter } 2 N \times 2 N i}=0
$$

where the $i$ represents the number of total CUs, $i=4^{n}, n \in[0,1,2,3], n$ is the quadtree depth level; $Q_{\text {Merge }_{i}}$ is equal to zero, if the prediction residuals of the Merge/Skip mode are quantized to zeros, 
otherwise, $Q_{\text {Merge }_{i}}$ is larger than zero; $Q_{\text {Inter } 2 \mathrm{~N} \times 2 \mathrm{~N} i}$ is equal to zero, if the prediction residuals of the inter- $2 \mathrm{~N} \times 2 \mathrm{~N}$ mode are quantized to zeros, otherwise, $Q_{\operatorname{Inter} 2 \mathrm{~N} \times 2 \mathrm{Ni}_{\mathrm{i}}}$ is larger than zero.

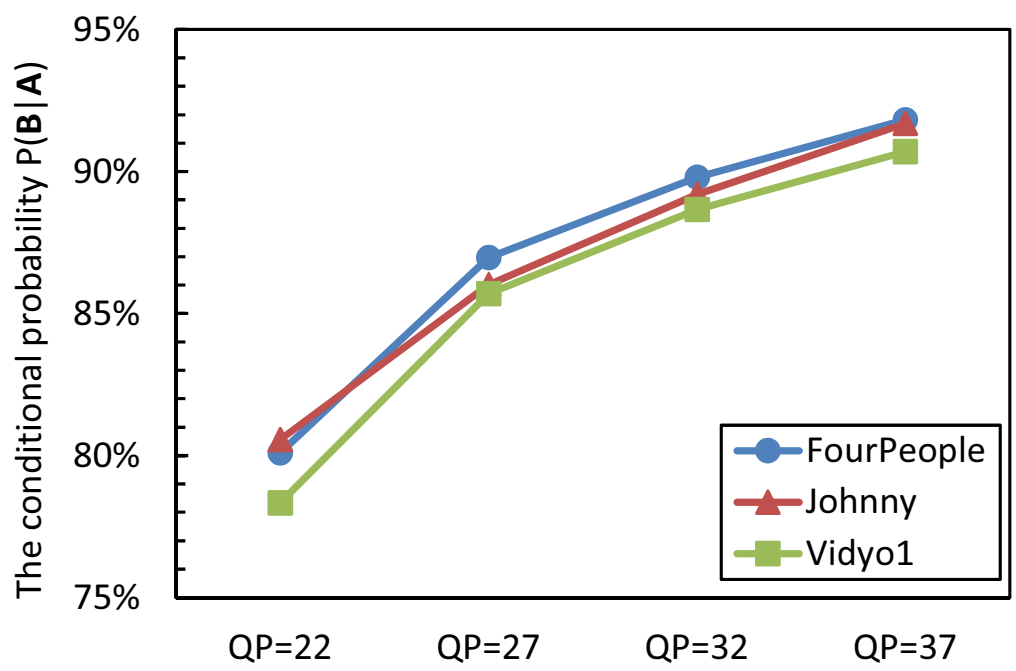

Figure 2. The conditional probability $\mathrm{P}(\mathbf{B} \mid \mathbf{A})$.

\subsection{Efficient Distortion Estimation Based on Spatial Correlation}

In the inter-prediction process, the distortion value is used to find the best matching block. The sum of absolute distortion (SAD) is a normal distortion calculation method in video coding; however, the distortion calculation process consumes the majority of the total encoding time [22]. If this process can be simplified, much more of the encoding time could be reduced. In the visual sensor videos, there exist a huge number of homogenous regions, such as the background. In this paper, the content property of one $\mathrm{CU}$ is determined according to the quantized coefficients; thus, the current encoding CU belongs to a homogenous region if:

$$
Q_{\text {Merge }}+Q_{\text {Inter } 2 N \times 2 N}=0
$$

where $Q_{\text {Merge }}$ denotes the quantized coefficients of the $\mathrm{CU}$, which is encoded with the Merge/Skip mode, if after encoding the $\mathrm{CU}$ with the Merge/Skip mode, the prediction residuals are transformed and quantized to zeros, then the value of $Q_{\text {Merge }}$ is equal to zero; $Q_{\text {Inter } 2 N \times 2 N}$ indicates the quantized coefficients of the $\mathrm{CU}$, which is encoded with the inter- $2 \mathrm{~N} \times 2 \mathrm{~N}$ mode; the value of $Q_{\text {Inter } 2 \mathrm{~N} \times 2 \mathrm{~N}}$ equals zero, if the prediction residuals are transformed and quantized to zeros after encoding the $\mathrm{CU}$ with the inter- $2 \mathrm{~N} \times 2 \mathrm{~N}$ mode.

Additionally, there is rather high distortion correlation in the spatial domain for these homogenous regions. Figure 3 shows an example of the spatial neighboring blocks of the current encoding block, where A, B and C represent the spatial neighboring blocks on the up, up-right and left of the current encoding block, respectively. Based on the spatial correlation [28,29], the motion vector of the current block, $M \vec{V}_{P r e d}$, is predicted by the spatial neighboring blocks and is given in Equation (4),

$$
M \vec{V}_{\text {Pred }}=\operatorname{Median}\left\{M \vec{V}_{\text {up }}, M V_{\text {up }- \text { right }}, M \vec{V}_{\text {left }}\right\}
$$

where $M \vec{V}_{\text {up }}, M V_{\text {up-right }}$ and $M \vec{V}_{\text {left }}$ indicate the motion vector of the neighboring blocks of up, up-right and left of the current block. Then, the predictive distortion for the $\mathrm{CU}$ with homogenous content, $S A D_{\text {Pred }}$, is obtained by:

$$
\operatorname{SAD}\left(o, c\left(M \vec{V}_{\text {Pred }}\right)\right)=\sum_{x=1}^{P_{x}} \sum_{y=1}^{P_{y}}\left|o(x, y)-c\left(x-M V_{\text {Pred }}, y-M V_{\text {Pred }}\right)\right|
$$


where $P_{x}$ and $P_{y}$ mean the prediction unit size; $o$ is the current value, and $c$ indicates the reconstructed block value; $M \vec{V}_{\text {pred }}=\left(\right.$ Pred $_{x}$, Pred $\left._{y}\right)$ is the predictive motion vector.

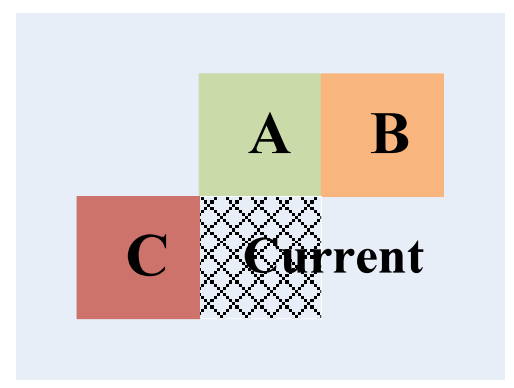

Figure 3. The spatial neighboring blocks of the current encoding block.

To evaluate the distortion estimation accuracy, the event $C$ represents that the current PU belongs to a homogenous region, and the event $\mathbf{D}$ denotes that the distortion that is obtained by Equations (4) and (5) is equal to the value that is gained by the original motion estimation. The conditional probability of $P(\mathbf{D} \mid \mathbf{C})$ is analyzed, and the results are shown in Figure 4.

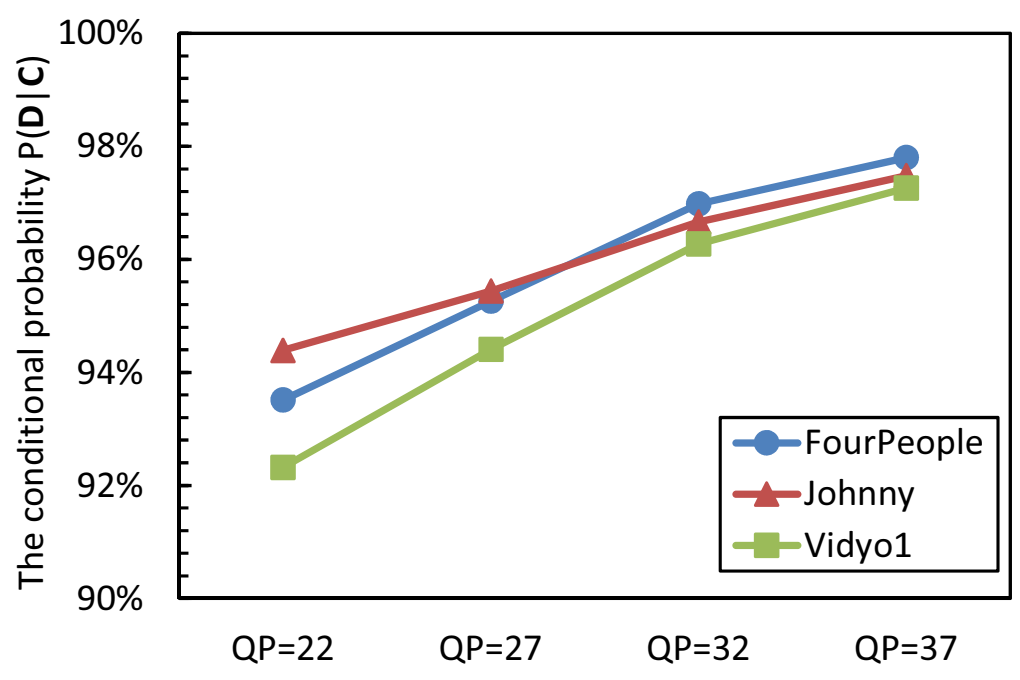

Figure 4. The conditional probability $\mathrm{P}(\mathbf{D} \mid \mathbf{C})$.

From Figure 4, it can be seen that the CU belongs to the homogenous region, and there is a large probability that the predictive distortion is the same as the distortion obtained by the original motion estimation. The probability of $P(\mathbf{D} \mid \mathbf{C})$ is from $92.32 \%$ to $97.80 \%, 95.65 \%$ on average. We can also see that with the increase of QP values, the value of $P(\mathbf{D} \mid \mathbf{C})$ becomes larger; this is because the large QP makes the coding content become homogenous. From these values, we can draw the conclusion that the proposed distortion estimation method can efficiently predict the distortion for a CU with homogenous content.

\subsection{The Overall Algorithm}

Based on the above analyses, the proposed fast CU size decision method for the low complexity HEVC encoder is summarized in Algorithm 1. 


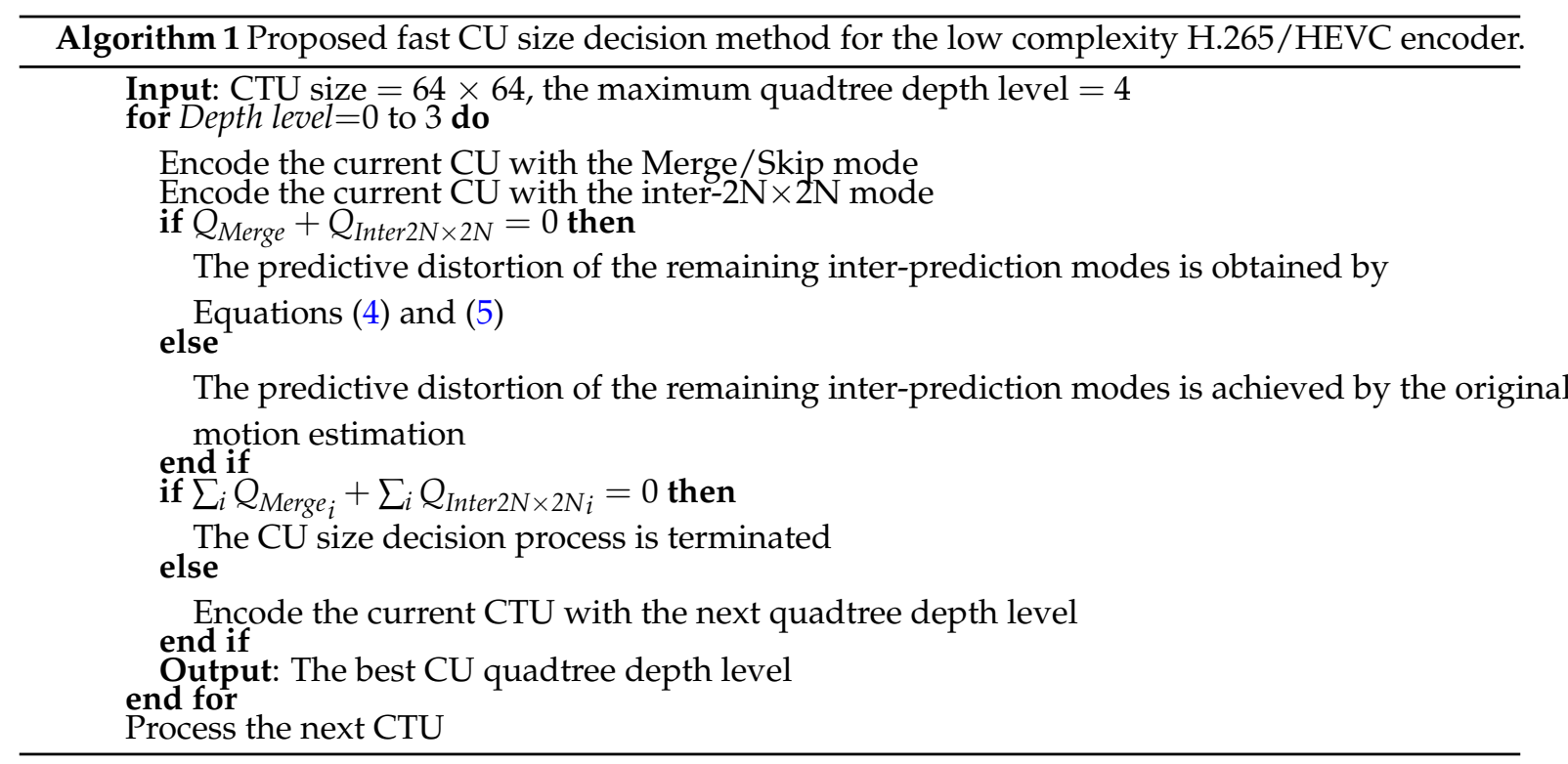

\section{Experimental Results}

To evaluate the efficiency of the proposed fast CU size decision method, the HEVC reference software HM12.0 is used as the software platform. The test conditions are listed as follows: the maximum CU size is $64 \times 64$; the maximum CU quadtree depth level is four; the motion estimation method is TZSearch, and the search range equals [-64, 64]; four QPs, 22, 27, 32 and 37, are used in our experiments. Six visual sensor video sequences, including "FourPeople", "Johnny", "KristenAndSara", "Vidyo1", "Vidyo3" and "Vidyo4", are adopted. These six video sequences are shown in Figure 5. The detailed information of these six sequences is that the resolution is $1280 \times 720$; the frame rate equals $60 \mathrm{fps}$; the number of encoded frames is 193 . The hardware platform is Intel Xeon CPU E3-1241 v3 with $3.50 \mathrm{GHz}$ and $3.50 \mathrm{GHz}, 4.00 \mathrm{~GB}$ RAM with the Microsoft Windows 7 64-bit operating system.

We compared the encoding performance of the proposed method with Choi [11], Kim [20] and Pan [12], in terms of peak signal-to-noise ratio (PSNR), bit rate (BR) and total encoding time savings. The experimental results are summarized in Table 2. In the table, $\Delta T$ means the total encoding CPU time savings, and this is computed as:

$$
\left\{\begin{array}{r}
\triangle P S N R=P S N R_{\Omega}-P S N R_{o}, \\
\Delta B R=\frac{B R_{\Omega}-B R_{o}}{B R_{o}} \times 100 \%, \\
\Delta T=\frac{T_{\Omega}-T_{o}}{T_{o}} \times 100 \%
\end{array}\right.
$$

where $P S N R_{\Omega}, B R_{\Omega}$ and $T_{\Omega}$ representing the PSNR, BR and total encoding time of method $\Omega$; $\Omega \in\{$ Choi, Kim, Pan $\}, P S N R_{o}, B R_{o}$ and $T_{o}$ indicate the PSNR, BR and total encoding time of the original HM12.0. In addition, the average $\triangle P S N R$ and $\triangle B R$ are computed by the Bjontegaard delta PSNR (BDPSNR) and Bjontegaard delta BR (BDBR) [30,31], respectively. 


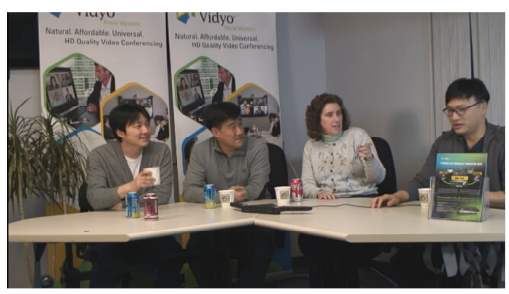

(a)

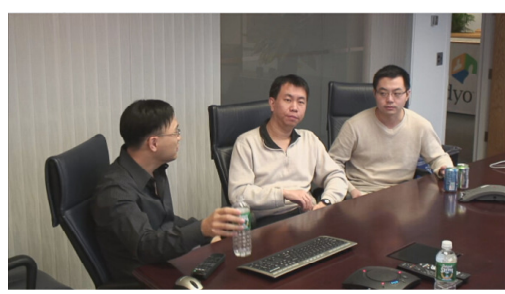

(d)

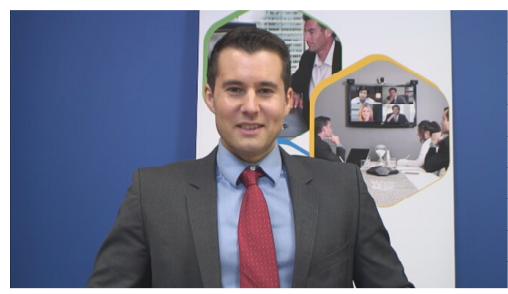

(b)

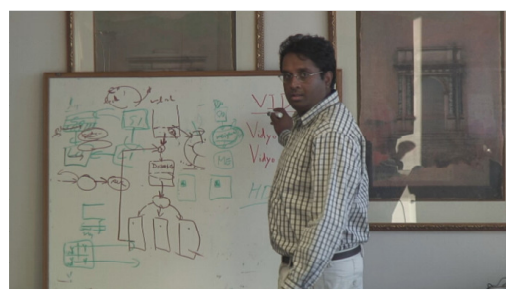

(e)

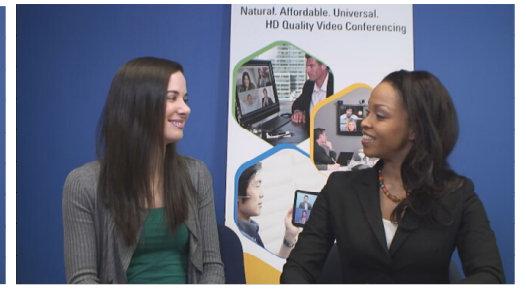

(c)

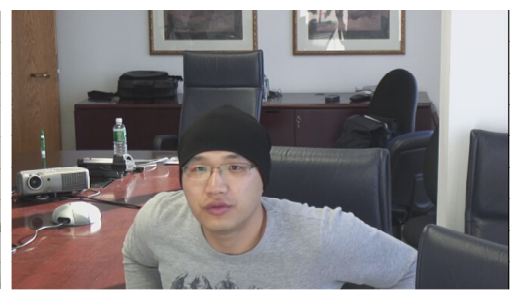

(f)

Figure 5. The test sequences. (a) FourPeople; (b) Johnny; (c) KristenAndSara; (d) Vidyo1; (e) Vidyo3; (f) Vidyo4.

From Table 2, it can be seen that Choi's method reduces the total encoding time from $32.54 \%$ to $70.17 \%, 55.47 \%$ on average. Meanwhile, the PSNR degrades from $0.022 \mathrm{~dB}$ to $0.071 \mathrm{~dB}$, and the BR increases from $-0.38 \%$ to $-1.99 \%$. The average PSNR variation is from $-0.003 \mathrm{~dB}$ to $0.033 \mathrm{~dB}$, $0.002 \mathrm{~dB}$ on average, and the average BR changes from $-0.98 \%$ to $0.70 \%, 0.07 \%$ on average. Kim's method saves the total encoding time from $29.06 \%$ to $56.22 \%, 45.71 \%$ on average. At the same time, the average PSNR change is from $-0.025 \mathrm{~dB}$ to $0.030 \mathrm{~dB},-0.005 \mathrm{~dB}$ on average, and the average $\mathrm{BR}$ variation is from $-0.90 \%$ to $0.68 \%, 0.18 \%$ on average. Pan's method improves the encoding complexity from $33.82 \%$ to $69.12 \%, 54.28 \%$ on average. Meanwhile, the average PSNR degrades from $0.009 \mathrm{~dB}$ to $0.031 \mathrm{~dB}, 0.018 \mathrm{~dB}$ on average, and the BR increases from $0.34 \%$ to $1.15 \%, 0.67 \%$ on average. The proposed method reduces the total encoding time from $51.27 \%$ to $85.26 \%, 71.91 \%$ on average. Moreover, the PSNR degrades from $0.030 \mathrm{~dB}$ to $0.114 \mathrm{~dB}$, and the BR variation is from $-1.43 \%$ to $0.70 \%$. The average PSNR change is from $-0.064 \mathrm{~dB}$ to $0.033 \mathrm{~dB},-0.021 \mathrm{~dB}$ on average, and the average $\mathrm{BR}$ variation is from $-1.02 \%$ to $1.98 \%, 0.73 \%$ on average. From these values, we can observe that the proposed method achieves a similar rate distortion performance as these compared methods, while the encoding complexity saving performance is the best among these compared methods.

To intuitively show the encoding time saving performance of the proposed method, the encoding time saving comparison among the Choi, Kim, Pan and proposed method is given in Figure 6. It can be observed that the proposed method obtains the best complexity saving performance. Compared to Chio's, Kim's and Pan's methods, the proposed method reduces $36.92 \%, 48.26 \%$ and $38.56 \%$ of the total encoding time, respectively. These values demonstrate that the proposed method works efficiently for reducing the encoding complexity of the HEVC encoder for VSNs. 
Table 2. Summary of the encoding results. BR, bit rate.

\begin{tabular}{|c|c|c|c|c|c|c|c|c|c|c|c|c|c|}
\hline \multirow[t]{2}{*}{ Sequence } & \multirow[t]{2}{*}{ QP } & \multicolumn{3}{|c|}{ Choi [11] vs. HM } & \multicolumn{3}{|c|}{ Kim [20] vs. HM } & \multicolumn{3}{|c|}{ Pan [12] vs. HM } & \multicolumn{3}{|c|}{ Proposed vs. HM } \\
\hline & & $\begin{array}{c}\triangle \text { PSNR } \\
\text { (dB) }\end{array}$ & $\begin{array}{c}\Delta \mathrm{BR} \\
(\%)\end{array}$ & $\begin{array}{l}\Delta \mathrm{T} \\
(\%)\end{array}$ & $\begin{array}{c}\Delta \text { PSNR } \\
\text { (dB) }\end{array}$ & $\begin{array}{c}\Delta \mathrm{BR} \\
(\%)\end{array}$ & $\begin{array}{l}\Delta \mathrm{T} \\
(\%)\end{array}$ & $\begin{array}{c}\Delta \text { PSNR } \\
\text { (dB) }\end{array}$ & $\begin{array}{r}\Delta \mathrm{BR} \\
\mathbf{( \% )}\end{array}$ & $\begin{array}{l}\Delta \mathrm{T} \\
(\%)\end{array}$ & $\begin{array}{c}\Delta \text { PSNR } \\
\text { (dB) }\end{array}$ & $\begin{array}{c}\Delta \mathrm{BR} \\
(\%)\end{array}$ & $\begin{array}{l}\Delta \mathrm{T} \\
(\%)\end{array}$ \\
\hline FourPeople & $\begin{array}{c}22 \\
27 \\
32 \\
37 \\
\text { Average }\end{array}$ & $\begin{array}{l}-0.030 \\
-0.039 \\
-0.025 \\
-0.029 \\
-0.004 \\
\end{array}$ & $\begin{array}{c}-1.32 \\
-1.28 \\
-0.65 \\
-0.62 \\
0.09\end{array}$ & $\begin{array}{l}-41.84 \\
-55.15 \\
-62.45 \\
-67.37 \\
-56.70 \\
\end{array}$ & $\begin{array}{c}-0.008 \\
-0.013 \\
0.001 \\
-0.012 \\
-0.002 \\
\end{array}$ & $\begin{array}{c}0.07 \\
-0.41 \\
-0.12 \\
0.04 \\
0.06\end{array}$ & $\begin{array}{l}-35.70 \\
-45.36 \\
-50.67 \\
-54.20 \\
-46.48 \\
\end{array}$ & $\begin{array}{l}-0.014 \\
-0.027 \\
-0.029 \\
-0.019 \\
-0.011 \\
\end{array}$ & $\begin{array}{c}-0.08 \\
0.15 \\
0.81 \\
-0.38 \\
0.34\end{array}$ & $\begin{array}{l}-39.27 \\
-52.35 \\
-60.81 \\
-66.34 \\
-54.69 \\
\end{array}$ & $\begin{array}{l}-0.034 \\
-0.041 \\
-0.047 \\
-0.063 \\
-0.021 \\
\end{array}$ & $\begin{array}{c}-1.13 \\
-0.99 \\
-0.57 \\
0.06 \\
0.61\end{array}$ & $\begin{array}{l}-58.04 \\
-71.59 \\
-78.38 \\
-82.46 \\
-72.62 \\
\end{array}$ \\
\hline Johnny & $\begin{array}{c}22 \\
27 \\
32 \\
37 \\
\text { Average }\end{array}$ & $\begin{array}{l}-0.024 \\
-0.030 \\
-0.046 \\
-0.039 \\
-0.003\end{array}$ & $\begin{array}{c}-1.22 \\
-1.16 \\
-1.21 \\
-1.30 \\
0.22\end{array}$ & $\begin{array}{l}-41.67 \\
-56.50 \\
-64.98 \\
-70.17 \\
-58.33 \\
\end{array}$ & $\begin{array}{l}-0.010 \\
-0.014 \\
-0.029 \\
-0.026 \\
-0.009\end{array}$ & $\begin{array}{c}-0.52 \\
-0.21 \\
-0.35 \\
-0.43 \\
0.44\end{array}$ & $\begin{array}{l}-36.45 \\
-46.92 \\
-52.46 \\
-56.22 \\
-48.01 \\
\end{array}$ & $\begin{array}{l}-0.019 \\
-0.016 \\
-0.042 \\
-0.021 \\
-0.008\end{array}$ & $\begin{array}{c}-0.32 \\
-0.57 \\
-0.53 \\
-0.38 \\
0.57\end{array}$ & $\begin{array}{l}-39.61 \\
-56.67 \\
-65.08 \\
-69.12 \\
-57.62 \\
\end{array}$ & $\begin{array}{l}-0.032 \\
-0.047 \\
-0.064 \\
-0.075 \\
-0.017\end{array}$ & $\begin{array}{c}-1.04 \\
-1.43 \\
-1.62 \\
-1.26 \\
0.69\end{array}$ & $\begin{array}{l}-58.17 \\
-74.11 \\
-81.09 \\
-85.26 \\
-74.66\end{array}$ \\
\hline KristenAndSara & $\begin{array}{c}22 \\
27 \\
32 \\
37 \\
\text { Average }\end{array}$ & $\begin{array}{l}-0.035 \\
-0.047 \\
-0.050 \\
-0.053 \\
-0.004\end{array}$ & $\begin{array}{c}-1.12 \\
-1.44 \\
-1.99 \\
-1.27 \\
0.01\end{array}$ & $\begin{array}{l}-41.23 \\
-54.02 \\
-62.69 \\
-67.84 \\
-56.45 \\
\end{array}$ & $\begin{array}{l}-0.010 \\
-0.014 \\
-0.013 \\
-0.012 \\
-0.012\end{array}$ & $\begin{array}{c}-0.28 \\
-0.09 \\
0.00 \\
0.19 \\
0.39\end{array}$ & $\begin{array}{l}-35.41 \\
-44.77 \\
-50.86 \\
-54.51 \\
-46.39 \\
\end{array}$ & $\begin{array}{l}-0.014 \\
-0.027 \\
-0.029 \\
-0.019 \\
-0.031\end{array}$ & $\begin{array}{c}-0.08 \\
0.15 \\
0.81 \\
-0.38 \\
1.15\end{array}$ & $\begin{array}{l}-39.27 \\
-52.35 \\
-60.81 \\
-66.34 \\
-54.69\end{array}$ & $\begin{array}{l}-0.047 \\
-0.072 \\
-0.079 \\
-0.089 \\
-0.019\end{array}$ & $\begin{array}{c}-1.08 \\
-2.35 \\
-1.59 \\
-0.84 \\
0.68\end{array}$ & $\begin{array}{l}-57.91 \\
-71.39 \\
-79.14 \\
-83.58 \\
-73.00\end{array}$ \\
\hline Vidyo1 & $\begin{array}{c}22 \\
27 \\
32 \\
37 \\
\text { Average } \\
\end{array}$ & $\begin{array}{c}-0.034 \\
-0.042 \\
-0.043 \\
-0.036 \\
0.033\end{array}$ & $\begin{array}{l}-1.40 \\
-1.28 \\
-1.06 \\
-1.40 \\
-0.98 \\
\end{array}$ & $\begin{array}{l}-43.63 \\
-55.67 \\
-62.99 \\
-68.12 \\
-57.60 \\
\end{array}$ & $\begin{array}{c}-0.003 \\
-0.013 \\
-0.024 \\
-0.022 \\
0.030 \\
\end{array}$ & $\begin{array}{c}0.21 \\
-0.22 \\
-0.46 \\
-0.76 \\
-0.90 \\
\end{array}$ & $\begin{array}{l}-36.67 \\
-45.59 \\
-50.79 \\
-54.25 \\
-46.82 \\
\end{array}$ & $\begin{array}{l}-0.014 \\
-0.026 \\
-0.027 \\
-0.023 \\
-0.009 \\
\end{array}$ & $\begin{array}{c}-0.36 \\
-0.82 \\
-0.40 \\
0.12 \\
0.29 \\
\end{array}$ & $\begin{array}{l}-40.42 \\
-52.61 \\
-61.17 \\
-65.18 \\
-54.84 \\
\end{array}$ & $\begin{array}{c}-0.042 \\
-0.056 \\
-0.063 \\
-0.070 \\
0.033\end{array}$ & $\begin{array}{l}-1.51 \\
-0.78 \\
-0.57 \\
-0.64 \\
-1.02 \\
\end{array}$ & $\begin{array}{l}-59.66 \\
-72.37 \\
-79.38 \\
-83.88 \\
-73.82 \\
\end{array}$ \\
\hline Vidyo3 & $\begin{array}{c}22 \\
27 \\
32 \\
37 \\
\text { Average } \\
\end{array}$ & $\begin{array}{l}-0.038 \\
-0.045 \\
-0.059 \\
-0.071 \\
-0.024 \\
\end{array}$ & $\begin{array}{c}-0.80 \\
-0.69 \\
-0.84 \\
-0.75 \\
0.70 \\
\end{array}$ & $\begin{array}{l}-40.47 \\
-51.98 \\
-59.36 \\
-65.03 \\
-54.21 \\
\end{array}$ & $\begin{array}{l}-0.010 \\
-0.020 \\
-0.041 \\
-0.017 \\
-0.025 \\
\end{array}$ & $\begin{array}{c}-0.16 \\
0.06 \\
-0.30 \\
0.69 \\
0.68 \\
\end{array}$ & $\begin{array}{l}-34.68 \\
-43.51 \\
-49.01 \\
-52.55 \\
-44.94 \\
\end{array}$ & $\begin{array}{l}-0.017 \\
-0.025 \\
-0.036 \\
-0.034 \\
-0.032 \\
\end{array}$ & $\begin{array}{c}-0.29 \\
-0.05 \\
0.52 \\
0.39 \\
1.04 \\
\end{array}$ & $\begin{array}{l}-39.41 \\
-51.86 \\
-60.94 \\
-66.03 \\
-54.56 \\
\end{array}$ & $\begin{array}{l}-0.053 \\
-0.061 \\
-0.114 \\
-0.112 \\
-0.064 \\
\end{array}$ & $\begin{array}{c}-1.11 \\
-0.40 \\
-0.39 \\
0.16 \\
1.98 \\
\end{array}$ & $\begin{array}{l}-57.07 \\
-69.79 \\
-76.91 \\
-81.96 \\
-71.43 \\
\end{array}$ \\
\hline Vidyo4 & $\begin{array}{c}22 \\
27 \\
32 \\
37 \\
\text { Average } \\
\end{array}$ & $\begin{array}{l}-0.029 \\
-0.022 \\
-0.026 \\
-0.028 \\
-0.009 \\
\end{array}$ & $\begin{array}{c}-0.69 \\
-0.72 \\
-0.38 \\
-0.40 \\
0.39 \\
\end{array}$ & $\begin{array}{l}-32.54 \\
-46.57 \\
-56.17 \\
-62.89 \\
-49.54 \\
\end{array}$ & $\begin{array}{l}-0.008 \\
-0.009 \\
-0.011 \\
-0.009 \\
-0.008 \\
\end{array}$ & $\begin{array}{c}-0.09 \\
-0.20 \\
0.29 \\
0.03 \\
0.38 \\
\end{array}$ & $\begin{array}{l}-29.06 \\
-39.92 \\
-46.56 \\
-50.81 \\
-41.59 \\
\end{array}$ & $\begin{array}{l}-0.015 \\
-0.024 \\
-0.016 \\
-0.017 \\
-0.018 \\
\end{array}$ & $\begin{array}{c}-0.26 \\
-0.14 \\
-0.03 \\
0.03 \\
0.60 \\
\end{array}$ & $\begin{array}{l}-33.82 \\
-47.37 \\
-55.37 \\
-60.62 \\
-49.29 \\
\end{array}$ & $\begin{array}{l}-0.030 \\
-0.037 \\
-0.041 \\
-0.049 \\
-0.036 \\
\end{array}$ & $\begin{array}{c}-0.72 \\
-0.58 \\
0.70 \\
0.46 \\
1.46\end{array}$ & $\begin{array}{l}-51.27 \\
-57.46 \\
-74.86 \\
-80.07 \\
-65.91 \\
\end{array}$ \\
\hline Average & & -0.002 & 0.07 & -55.47 & -0.005 & 0.18 & -45.71 & -0.018 & 0.67 & -54.28 & -0.021 & 0.73 & -71.91 \\
\hline
\end{tabular}

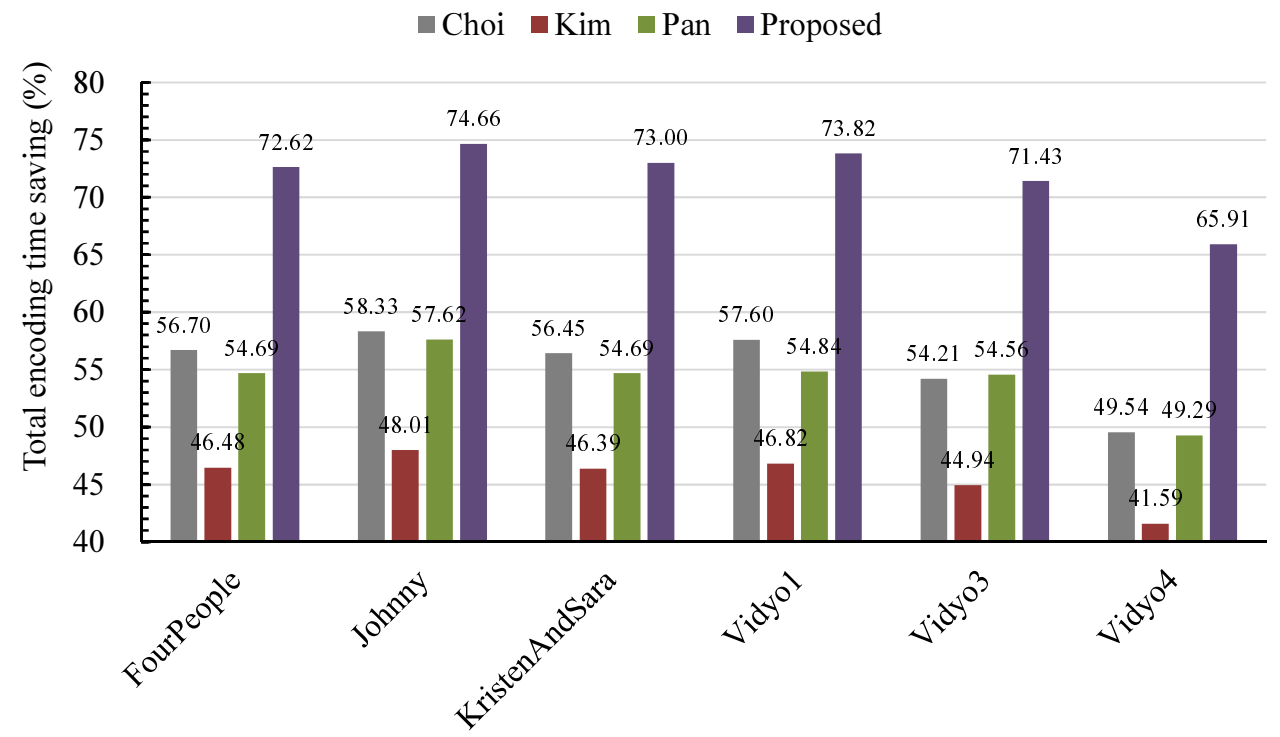

Figure 6. Comparison of the total encoding time savings.

\section{Conclusions}

High computational complexity becomes a bottleneck for the HEVC encoder to be used in VSNs. In order to reduce the encoding complexity of the HEVC encoder, in this paper, we proposed a low complexity HEVC CU size decision method based on the quantized coefficients, which consists of a CU depth early termination method and a low complexity distortion calculation method. Experimental results show that the proposed method can efficiently reduce the encoding complexity of the HEVC encoder of VSNs. 
Acknowledgments: This work was supported in part by the National Natural Science Foundation of China under Grant 61501246 and Grant 61232016, in part by the National Natural Science Foundation of Jiangsu Province of China under Grant BK20150930, in part by the Natural Science Foundation of the Jiangsu Higher Education Institutions of China under Grant 15KJB510019, in part by the Project through the Priority Academic Program Development of Jiangsu Higher Education Institutions, in part by the Startup Foundation for Introducing Talent of Nanjing University of Information Science and Technology (NUIST) under Grant 2243141501012 and in part by the Natural Science Foundation of Hebei Province of China under Grant F2015202311.

Author Contributions: All authors contributed significantly to the preparation of this manuscript. Zhaoqing Pan is the main author who proposed the idea, performed the experiments and wrote the manuscript. Liming Chen performed the experimental data processing and revised the manuscript. Xingming Sun discussed with the main author the proposed method and revised this manuscript.

Conflicts of Interest: The authors declare no conflict of interest.

\section{References}

1. Soro, S.; Heinzelman, W. A survey of visual sensor networks. Adv. Multimed. 2009, 2009, 1-21.

2. Costa, D.G.; Silva, I.; Guedes, L.A.; Vasques, F.; Portugal, P. Availability issues in wireless visual sensor networks. Sensors 2014, 14, 2795-2821.

3. Charfi, Y.; Wakamiya, N.; Murata, M. Challenging issues in visual sensor networks. IEEE Wirel. Commun. 2009, 2, 44-49.

4. Chung, Y.; Lee, S.; Jeon, T.; Park, D. Fast Video Encryption Using the H.264 Error Propagation Property for Smart Mobile Devices. Sensors 2015, 15, 7953-7968.

5. Costa, D.G.; Guedes, L.A. A survey on multimedia-based cross-layer optimization in visual sensor networks. Sensors 2011, 11, 5439-5468.

6. Xie, S.; Wang, Y. Construction of tree network with limited delivery latency in homogeneous wireless sensor networks. Wirel. Pers. Commun. 2014, 1, 231-246.

7. Sullivan, G.J.; Ohm, J.-R.; Han, W.-J.; Wiegand, T. Overview of the high efficiency video coding (HEVC) standard. IEEE Trans. Circuits Syst. Video Technol. 2012, 12, 1649-1668.

8. ISO/IEC 23008-2:2013 ITU-T Rec. H.265: Information technology-High Efficiency Coding and Media Delivery in Heterogeneous Environments-Part 2: High Efficiency Video Coding; ISO: Geneva, Switzerland, 2013.

9. Pan, Z.; Kwong, S. A direction-based unsymmetrical-cross multi-hexagon-grid search algorithm for H.264/AVC motion estimation. J. Signal Process. Syst. 2013, 7, 59-72.

10. Lei, J.; Feng, K.; Wu, M.; Li, S.; Hou, C. Rate control of hierarchical B prediction structure for multi-view video coding. Multimed. Tools Appl. 2014, 72, 825-842.

11. Choi, K.; Park, S.-H.; Jang, E.S. Coding tree pruning based CU early termination. In Proceedings of the ITU-T/ISO/IEC Joint Collaborative Team on Video Coding (JCT-VC) Document JCTVC-F092, Torino, Italy, 14-22 July 2011.

12. Pan, Z.; Kwong, S.; Sun, M.-T.; Lei, J. Early MERGE mode decision based on motion estimation and hierarchical Ddepth correlation for HEVC. IEEE Trans. Broadcast. 2014, 62, 405-412.

13. Pan, Z.; Kwong, S.; Zhang, Y.; Lei, J.; Yuan, H. Fast coding tree unit depth decision for high efficiency video coding. In Proceedings of the IEEE International Conference on Image Processing(ICIP), Paris, France, 27-30 October 2014; pp. 3214-3218.

14. Shi, H.; Fan, L.; Chen, H. A fast CU size decision algorithm based on adaptive depth selection for HEVC encoder. In Proceedings of the 2014 International Conference onAudio, Language and Image Processing (ICALIP), Shanghai, China, 7-9 July 2014; pp. 143-146

15. Zhang, Y.; Kwong, S.; Wang, X.; Yuan, H.; Pan, Z.; Xu, L. Machine learning-based coding unit depth decisions for flexible complexity allocation in high efficiency video coding. IEEE Trans. Image Process. 2015, $24,2225-2238$.

16. Ahn, S.; Lee, B.; Kim, M. A novel fast CU encoding scheme based on spatiotemporal encoding parameters for HEVC inter coding. IEEE Trans. Circuits Syst. Video Technol. 2015, 25, 422-435.

17. Goswami, K.; Kim, B.; Jun, D.; Jung, S.; Choi, J. Early coding unit-splitting termination algorithm for High Efficiency Video Coding (HEVC). ETRI J. 2014, 36, 407-417.

18. Tian, G.; Goto, S. Content adaptive prediction unit size decision algorithm for HEVC intra coding. In Proceedings of the 2012 Picture Coding Symposium (PCS), Krakow, Poland, 7-9 May 2012; pp. 405-408. 
19. Kim, M.; Ling, N.; Song, L.; Gu, Z. Fast skip mode decision with rate-distortion optimization for high efficiency Video coding. In Proceedings of the 2014 IEEE International Conference on Multimedia and Expo Workshops (ICMEW), Chengdu, China, 14-18 July 2014; pp. 1-6.

20. Kim, J.; Yang, J.; Won, K.; Jeon, B. Early determination of mode decision for HEVC. In Procedings of the 2012 Picture Coding Symposium (PCS), Krakow, Poland, 7-9 May 2012; pp. 449-452.

21. Lee, H.; Shim, H.J.; Park, Y.; Jeon, B. Early Skip mode decision for HEVC encoder with emphasis on coding quality. IEEE Trans. Broadcast. 2015, 61, 388-397.

22. Ohm, J.-R.; Sullivan, G.J.; Schwarz, H.; Tan, T.K.; Wiegand, T. Comparison of the coding efficiency of video coding standards-including High Efficiency Video Coding (HEVC). IEEE Trans. Circuits Syst. Video Technol. 2012, 12, 1669-1684.

23. Bossen, F.; Flynn, D.; Suehring, K. JCT-VC AHG Report: HEVC HM Software Development and Software Technical Evaluation (AHG3); (JCT-VC) Document JCTVC-O0003; ITU-T/ISO/IEC Joint Collaborative Team on Video Coding: San Jose, CA, USA, 2013.

24. Pan, Z.; Lei, J.; Zhang, Y.; Yan, W.; Kwong, S. Fast transform unit depth decision based on quantized coefficients for HEVC. In Proceedings of the 2015 IEEE International Conference on Systems, Man, and Cybernetics (SMC), Hong Kong, China, 9-12 September 2015; pp. 1127-1132.

25. Ma, L.; Li, S.; Ngan, K.N. Reduced-reference image quality assessment in reorganized DCT domain. Signal Process. Image Commun. 2013, 28, 884-902.

26. Ma, L.; Ngan, K.N.; Zhang, F.; Li, S. Adaptive block-size transform based just-noticeable difference model for images/videos. Signal Process. Image Commun. 2011, 26, 162-174.

27. Ma, L.; Li, S.; Zhang, F.; Ngan, K.N. Reduced-reference image quality assessment using reorganized DCT-based image representation. IEEE Trans. Multimed. 2011, 13, 824-829.

28. Chen, Z.; Xu, J.; He, Y.; Zheng, J. Fast inter-pel and fractional-pel motion estimation for H.264/AVC. J. Vis. Commun. Image Represent. 2007, 17, 264-290.

29. Pan, Z.; Kwong, S.; Xu, L.; Zhang, Y.; Zhao, T. Predictive and distribution-oriented fast motion estimation for H.264 / AVC. J. Real-Time Image Proc. 2014, 4, 597-607.

30. Bjontegaard, G. Calculation of Average PSNR Differences between RD-Curves. In Proceedings of the ITU-T Video Coding Experts Group (VCEG) Thirteenth Meeting, Austin, TX, USA, 2-4 April 2001.

31. Pan, Z.; Zhang, Y.; Kwong, S. Efficient motion and disparity estimation optimization for low complexity multiview video coding. IEEE Trans. Broadcast. 2015, 61, 166-176.

(C) 2015 by the authors; licensee MDPI, Basel, Switzerland. This article is an open access article distributed under the terms and conditions of the Creative Commons by Attribution (CC-BY) license (http://creativecommons.org/licenses/by/4.0/). 